\title{
La Evolución de los Derechos de la Infancia: Una Visión Internacional
}

\author{
Paulí Dávila Balsera \\ Luis María Naya Garmendia \\ Universidad Complutense de Madrid
}

\begin{abstract}
During the 20th century a process of internationalisation concerning children's rights has been operationalaized via, among other events, International Congresses held to exchange experiences and common protection policies between some European and American countries; and the emergence and application of national protection policies. In addition, working from the promulgation of three important international treaties in 1924, 1959 and 1989, these policies have expanded and are being used as a model by countries that are implementing them progressively. However, it can be proven that discourse on childhood in this period has been continuous, highlighting the rights to autonomy and above all those regarding protection and help for boys and girls in social exclusion situations.
\end{abstract}

Key words: Children's rights, History of Education, International Law.

RESUMEN

A lo largo del Siglo XX se aprecia un proceso de internacionalización de los derechos de la infancia a partir, entre otros acontecimientos, de la celebración de Congresos Internacionales que dieron lugar a un intercambio de experiencias y de políticas de protección comunes entre algunos países de Europa y América; además del surgimiento y aplicación de políticas protectoras a nivel nacional. Asimismo, y a partir de la promulgación de tres importantes tratados internacionales en 1924, 1959 y 1989, esas políticas se expanden, siendo utilizadas como modelo por parte de países que las irán implementando paulatinamente. No obstante, se puede constatar que el discurso sobre la infancia en ese período se mantiene permanente, destacando los derechos de autonomía y, sobre todo, los de protección y ayuda a los niños y niñas en situaciones de exclusión social.

Descriptores: Derechos de la Infancia, Historia de la Educación, Derecho Internacional.

RÉSUMÉ

Tout au long du XXè siècle on observe un processus d'internationalisation des droits de l'enfance à partir, entre autres, de la célébration de Congrès Internationaux qui ont donné lieu à une échange d'expériences et de politiques de protection communes entre certains pays d'Europe et d'Amérique ; à ceci il faut ajouter l'apparition et la mise en marche de politiques protectrices au niveau national. Aussi, et à partir de la promulgation de trois traités internationaux de grande importance, en 1924, 1959 et 1989, ces politiques se répandent et sont utilisées comme modèle 
par des pays qui les mettront en oeuvre au fur et à mesure. Cependant, on remarque que le discours sur l'enfance en cette période persiste, l'accent étant mis sur les droits d'autonomie et surtout ceux de protection et d'aide aux enfants dans des situations d'exclusion sociale.

Mots-clés: Droits de l'enfance, Histoire de l'éducation, Droit international.

\section{Introducción ${ }^{1}$}

$\mathrm{H}$ ablar, en la actualidad, de los derechos de los niños y las niñas no es nada extraño. Es más, existe cierta familiarización con el tema debido a los casos, cada vez más visibles, de violación de estos derechos. Pero hace un siglo hablar de este tipo de derechos era prácticamente imposible, lo cual no quiere decir que no se protegiese a la infancia de las situaciones de desamparo en las que vivía, o se legislara para mejorar su situación. Lo que ha ocurrido es que a finales del siglo XX, la Asamblea General de las Naciones Unidas aprueba la Convención sobre los Derechos del Niño, recogiendo una tradición existente sobre esos derechos y que está teniendo amplia repercusión en las políticas sociales sobre la infancia.

En este artículo vamos a tratar de analizar el paulatino proceso de reconocimiento de los derechos del niño y la niña. Proceso histórico que hizo posible que se descubriera, en un momento determinado, que los niños son seres humanos con derechos y que deben ser reconocidos internacionalmente. En este sentido, y en primer lugar, estudiaremos ese proceso de internacionalización a partir de una serie de congresos sobre protección a la infancia que tuvieron lugar durante el primer tercio del siglo XX, por entender que pusieron las bases para las posteriores políticas internacionales; en segundo lugar, daremos cuenta de la articulación de los diferentes tratados internacionales; y en tercer lugar analizaremos el discurso proteccionista subyacente en los mismos. Por lo tanto, nos interesa resaltar lo siguiente: primero, que a lo largo de los siglos XIX y XX existe una serie de políticas nacionales de protección a la infancia ${ }^{i i}$; segundo, que se da un progresivo proceso de internacionalización de estas políticas, a partir de la creación de asociaciones y congresos sobre protección a la infancia; tercero, que la asunción por parte de organismos internacionales de los derechos de la infancia, a través de diferentes tratados desde 1924 a 1989, ha supuesto un fenómeno expansivo, proponiendo unos modelos de aplicación a los países en desarrollo y cuarto, que el discurso que sostienen los tratados y documentos sobre los derechos de la infancia no ha variado sustancialmente hasta la Convención de 1989, donde por primera vez, se reconocen unos derechos de civiles a los niños y niñas, además de los clásicos de protección y ayuda.

Desde la perspectiva actual, nos interesa resaltar que esas políticas nacionales, al igual que su internacionalización, han supuesto el reconocimiento de los derechos de los niños y niñas que se fragua a lo largo del siglo XX, suponiendo un cambio en su consideración jurídica. Los niños habrían pasado a ser sujetos de derecho, como cualquier adulto, en contra de la consideración tradicional de los niños como objetos de derecho (Hierro, 1999). Esta situación permite hablar de una evolución en cuanto a la consideración autónoma del niño y, por lo tanto, añade algo nuevo a la tradicional postura de hablar de la protección a la infancia. El desarrollo internacional de los derechos del niño muestra que "desde las declaraciones de 1924 y 1959 hasta el Convenio 
(sic) de los Derechos del Niño de 1989, las concepciones sobre el fundamento de los derechos del menor y el papel del Estado han cambiado" (Calvo, 2003: 156). De manera que este último tratado supone un punto de inflexión en el discurso sobre la infancia, al conjugar las diversas trayectorias políticas sobre la infancia.

\section{Los congresos de protección a la infancia y su perspectiva internacional}

Como se está poniendo de manifiesto en la historiografía reciente sobre la infancia, existen diferentes formas de acercarse al conocimiento de los niños y niñas desde la perspectiva histórica. Una de ellas es el estudio de la protección infantil, de forma que en la actualidad contamos con numerosas obras que abordan esta temática, sobre todo en los países industrializados de Europa y América, pudiendo afirmarse que cada Estado ha seguido sus propias dinámicas para desarrollar sus políticas de protección y ayuda a la infancia, especialmente durante los siglos XIX y XX $\mathrm{XX}^{3}$. Hay que resaltar que se trata de políticas protectoras llevadas a cabo por los diferentes países occidentales, que viven los mismos "problemas" y aplican parecidas soluciones. No obstante, podemos apreciar también que, a pesar de las tradiciones culturales, religiosas, económicas, sociales y educativas, existe un cierto acuerdo en aplicar las mismas políticas aunque con una cierta distancia temporal entre los diversos países. De esta manera, podemos afirmar que existe un cierto acuerdo en las "soluciones" a tomar. Que ello sea así se debe a los procesos de industrialización que se estaban llevando a cabo y a sus consecuencias sobre la infancia y las familias y, también, a los agentes sociales, religiosos y educativos que intervenían para paliar la situación de la infancia.

Pero, sin duda, uno de los elementos más llamativos, en cuanto a este tipo de prácticas, es la transición a los ámbitos internacionales de esas políticas protectoras llevadas a cabo por los diferentes Estados nacionales. La celebración de congresos, las relaciones gubernamentales y los tratados de carácter internacional van a favorecer este tipo de dinámica, debido a los nuevos contextos mundiales y, posteriormente, al surgimiento de organismos internacionales, a raíz de las dos guerras mundiales. Tanto la Sociedad de Naciones, como su sucesora las Naciones Unidas, se convirtieron en marcos donde, además de atender su objetivo principal -evitar una nueva guerra y trabajar por los objetivos de la paz internacional- se elaboraron toda una serie de tratados en los que prevalecen los derechos humanos. En este proceso de transición, y también de interacción, se fragua, como indica Rollet (2001) "una cultura común" a partir de los congresos internacionales. En ese periodo muchos Estados participaron en los debates sobre la definición del niño, las formas de protegerlo, el trabajo infantil o el papel de la educación, al igual que tantas sociedades médicas, educativas o de higiene, debido sobre todo a la existencia de un problema clave desde el siglo XIX como era la mortalidad infantil y las condiciones económicas en las que vivían muchas familias. De forma que, incluso la constitución del concepto moderno del menor, como señala Therborn (1993:87), tiene estas fuentes de definición: "El derecho y las distintas formas de intervención del estado jugaron un papel crucial en la constitución del concepto moderno de menor. Las dos fuentes fundamentales determinantes en el establecimiento del con- 
cepto de minoría de edad han sido las legislaciones relativas la educación obligatoria y las que regulan las condiciones de trabajo".

Desde finales del siglo XIX y a lo largo del primer tercio del siglo XX se produce un fenómeno que se irá consolidando, en relación con las políticas de protección infantil. Se trata del progresivo interés por parte de los reformadores sociales, médicos, pedagogos, educadores, pediatras, asociaciones de protección a la infancia y, en general, por los gobiernos, de internacionalizar dichas políticas. Una de las vías de penetración será la celebración de congresos internacionales que, en la mayoría de los casos, están convocados por asociaciones de protección a la infancia, pediatría, higiene, medicina, etc. y que cuentan con el patrocinio de gobiernos nacionales. Es cierto que la mayoría de estos congresos científico-sociales tiene un carácter propagandístico, planteándose la lucha contra enfermedades extendidas en la época o alertando sobre problemas sociales relacionados con las condiciones de la infancia; pero también, su objetivo era extender estos problemas a la sociedad, de manera que contribuyesen a crear una cierta presión ideológica que permitiera la intervención práctica. En este sentido, la presencia de autoridades políticas y de la elite científica permitía ciertas garantías de éxito, aunque nada más fuese en las recomendaciones que, generalmente, se hacían para tomar medidas prácticas de intervención política.

Las discusiones en los congresos sobre la infancia giraban alrededor de dos polos "un polo más jurídico y teórico y otro más pragmático" (Rollet; 2001: 99). Es decir, un planteamiento jurídico más preocupado por cuestiones relativas a las responsabilidades de los Estados o de las familias con respecto a los niños abandonados, la delincuencia o la tutela. En este ámbito los cambios de la legislación adquieren una gran importancia. Todas estas cuestiones, por ejemplo, se pueden observar ya en 1883 a raíz del primer Congreso Internacional de Protección a la Infancia, celebrado en Paris. O bien un planteamiento médico-higienista más preocupado por la primera infancia y el cuidado de las enfermedades más comunes, además de la apertura al campo de la puericultura y a la introducción de la leche esterilizada, como puede observarse en los diferentes congresos sobre Higiene. En este segundo ámbito, más pragmático, es de resaltar la celebración de los tres Congresos Internacionales de Gotas de Leche (París, 1905; Bruselas, 1907 y Berlín 1911) que tuvieron un éxito sin precedentes, tanto por el importante número de países que participaron, como por la presencia de pediatras de alto nivel científico. Los países que tuvieron mayor presencia en los tres congresos fueron los siguientes: Francia, Alemania, Bélgica, Reino Unido, España, Italia, Argentina, Holanda y Suiza. Las discusiones que se llevaron a cabo, por ejemplo, en el primer congreso, versaban sobre los depósitos de leche o la distribución de leche asociada a la visita médica, pero en el segundo y tercer congreso los temas se ampliaron, de manera que se abordaron temas que iban desde la educación, la legislación de protección a la infancia o la estadística sobre mortalidad infantil (Rollet, 2001: 104-106). Por supuesto, estos congresos tuvieron sus consecuencias en los diferentes países con la creación de este tipo de instituciones.

Estos congresos, además de las publicaciones, libros, folletos divulgativos y otro tipo de material, permitían dar cierta resonancia a determinados eventos más o menos científicos. En este sentido, un repaso por los congresos internacionales celebrados a finales 
del siglo XIX y en el primer tercio del siglo XX, nos permite observar los agentes implicados, los temas de interés, la resonancia social, las implicaciones políticas y en, cierta manera, la situación de la infancia en ese periodo. Además, esa preocupación porque los congresos fuesen internacionales, nos manifiesta también otra dimensión nueva: constatar que los problemas y las soluciones que parecen característicos de un determinado país no lo eran, que las causas de exclusión social en la que vivían tantas mujeres y niños en Europa, y en el mundo, era un patrimonio compartido. A partir de la constatación de estas realidades, se irá fraguando una red de relaciones y asociaciones internacionales, con sus respectivos comités nacionales y organismos interdependientes que favorecerán en cierta medida una nueva consideración de la infancia y también, por primera vez, convertir a la infancia en un objeto específico de diversos tratados internacionales.

En la mayoría de los casos, los temas objeto de discusión de estos congresos internacionales solía coincidir con las disposiciones legislativas que sobre la infancia se estaban desarrollando en diversos países europeos y americanos. Como puede observarse la muestra de intereses es amplia en cuanto se recoge todo tipo de congresos, no solo los relacionados con la protección específicamente, sino otros que tienen que ver con la higiene, la educación familiar, pediatría, tribunales para niños, colonias de vacaciones, asistencia pública, etc. ${ }^{4}$. En esta amalgama de intereses es donde se construye esa "cultura común" de la que habla Rollet y también donde se marcan los límites y posibilidades del discurso sobre la infancia. Entre los países con mayor capacidad de convocatoria figura en primer lugar, y de manera muy destacada, Francia, seguida de Bélgica -con un 50 por 100 menos de congresos-, Italia, España, Estados Unidos, Suiza, Alemania, Inglaterra, Argentina, Hungría, Suecia, Brasil, y otros países latinoamericanos debido a la celebración de los congresos panamericanos del niño ${ }^{5}$. También tendríamos que sumar todos los congresos nacionales celebrados en diversos países sobre tribunales tutelares de menores (Alemania, 1913), pornografía infantil (Francia, 1911), colonias de niños (Inglaterra, Francia, 1911), etc.

Al igual que ocurre con los congresos estudiados por Rollet, y que corresponden al periodo comprendido entre 1883 y 1902, se constata que entre 1909 y 1930, los dos polos citados anteriormente, continúan manteniéndose. Es decir, por una parte, los congresos centrados en los ámbitos jurídicos o de aspecto más social (donde podemos incluir los relativos a diferentes aspectos educativos, a los tribunales tutelares de menores, a la justicia del menor, a la pornografía infantil, o al alcoholismo); y el otro polo relacionado con aspectos más pragmáticos y médico-higiénicos (con temas sobre pediatría, medicina, higiene, eugenesia, salud o gotas de leche). Por lo tanto, una clasificación posible del contenido de estos congresos tendría que tener en cuenta estos dos grandes ámbitos de la protección infantil. No obstante, no resulta extraño que así sea y que, además, se haya mantenido durante más de un siglo, pues básicamente recoge los dos capítulos más sobresalientes de la protección: el derecho a la vida y el derecho a la protección y ayuda. Así mismo, a lo largo de todo el periodo estudiado, se observa que existe una sustitución de modelos en la protección a la infancia, de manera que en el último periodo se va imponiendo el modelo americano y anglosajón, donde la protección se relaciona con la situación de la madre, la educación y el rol de la mujeres; en 
contra del modelo anterior, dominado por Francia y la Europa latina, más preocupada por el cuerpo de los niños, sus enfermedades y la atención a su salud. Aspectos que se pueden encontrar no solamente en los congresos, sino también en las políticas de protección.

Por otra parte, las asociaciones internacionales también tendrán su parte importante en la organización de este tipo de congresos, que se irán celebrando con cierta periodicidad. Entre las más importantes, podemos destacar la Asociación Internacional para la Protección de la Infancia, la Unión Internacional para la protección de la Infancia en la primera edad, La Unión Internacional de Socorros de Niños y la Liga de Sociedades de la Cruz Roja. Estas cuatro asociaciones lograron colaborar conjuntamente en la celebración del Congreso Internacional de Protección a la Infancia, celebrado en julio de 1928, en París, y al cual asistieron más de 2000 delegados extranjeros. La Unión Internacional para la Protección de la Infancia en la primera edad surgió a propuesta del Dr. Eugenio Lust, promotor del primer Congreso de Gotas de Leche celebrado en París en 1902 y que conseguiría la participación económica de los Estados adheridos, además de una oficina internacional en Bruselas. Esta asociación reelaborará en 1948 la Declaración de Ginebra de 1924, a la que nos referiremos más adelante, y entre sus miembros se encontraban importantes personajes del mundo de la medicina. En su proyecto de estatuto, elaborado en 1914, se cita como objeto de la misma "servir de lazo de unión entre todos los que en diferentes países se interesan por la protección de la Infancia" con la finalidad de favorecer el progreso de las legislaciones, "así como la conclusión de pactos internacionales sobre la materia”. El Boletín Internacional de la Protección de la Infancia sería el órgano para intercambiar sus informaciones y la sede del Comité internacional se situaría en Bruselas (Pro-Infantia, tomo VI, 1914: 174).

Además de estas importantes asociaciones podemos observar, a través de Pro-Infantia, el surgimiento de otra serie de asociaciones, que sorprende por su elevado número y el ámbito al que se dedican. Según una noticia recogida por la revista Independent de Estados Unidos, en este país en 1911 existían 350 sociedades de protección a la infancia, la primera de ellas se fundó en 1876 (Pro-Infantia, tomo III, 1911: 140). Así, surgirán unas asociaciones dedicadas al humanitarismo (Obra de la Sociedad Humanitaria de Milán, en 1905), a la mortalidad infantil que ya existía en Francia a finales del siglo XIX (Sociedad contra la mortalidad infantil en Estados Unidos), o sobre niños "anormales" (Asociación suiza de los institutores (sic) de niños anormales, creada en 1911), Sociedad Nacional de Amigos de la Infancia; Instituto de Puericultura, Sociedades de Caridad maternal en La Vendée, creadas en 1909, Liga francesa de la educación moral, Sociedad Femenina de la Asistencia Pública, fundada en Argentina en 1823, Sociedad Francesa de Eugénica de 1913, Unión francesa para la corrección de la infancia, Sociedad de Nueva York para la protección de los niños, Federación de amigos y protectores de la Infancia en Brasil de1924, Sociedad alemana de protección a la infancia, Liga de Educación familiar en Bélgica, 1913; Sociedad pediátrica de Madrid, Asociación matritense de Caridad, Asociación de estudios penitenciarios y rehabilitación del delincuente, de Madrid. También surge la sorprendente Sociedad Protectora de niños, pájaros y plantas de Argentina, que en 1915 proponía "alejar a la niñez poco a poco del corruptor deletéreo cinematógrafo” (Pro-Infantia, tomo X, 1915: 229). En 1916, en plena primera guerra 
mundial, en Francia funcionaban las siguientes obras protectoras: La Liga Fraternal de los hijos de Francia, el Patronato de la Infancia, la Obra del Recurso, la tutelas, la Obra de Adopción y numerosos asilos gestionados por las Hijas de la Caridad; además del Orfanato de Huérfanos, la Asociación Nacional para la protección de viudas y huérfanos de la guerra, la Asociación Nacional francesa para la protección de las familias de los muertos por la Patria, la Asociación de huérfanos de guerra, los pupilos de la escuela y otras más (Pro-Infantia, tomo XIII, 1917: 352). En Rumania, en 1930, la princesa Alejandra Cantacuzene, vicepresidenta del Consejo Internacional de las Mujeres, presentó a la Asamblea de Higiene de la Liga de las Naciones el proyecto de un Instituto Internacional de la Infancia encabezado por una "llamada a las mujeres de todas las naciones (ProInfantia, Año XXII, 1930: 280). Es decir, con toda esta información se puede elaborar un amplio inventario de este tipo de asociaciones nacionales, además de las internacionales, que en nada envidian a las actuales ONG. Entre ellas existían relaciones de diverso tipo tanto personal como orgánico.

En este contexto de interés internacional por la protección de los niños y niñas, y en plena primera guerra mundial en 1917 , tenemos conocimiento de un proyecto de "carta magna internacional" propuesta por la Liga internacional para la protección a la Infancia en los Estados Unidos de América, con sede en Nueva York, cuyo objetivo era paliar la situación de los niños en Europa y cuyo trabajo fundamental sería "asegurar la cooperación de todas las Naciones, por medio de sus legisladores y de sus hombres más eminentes, en la Iglesia, en el estado, en el ejercito y en la Marina, para la gran obra de reconstrucción que ha de seguir la paz en Europa" para ello se propone esta carta magna, donde de manera explícita se habla de reconocer "una amplia relación de derechos del niño, que debe nacer en buenas condiciones, que debe ser educado y protegido contra la indiferencia y la ignorancia y la codicia" y cuyo código "de los derechos del niño debe exigirse como parte integral entre las condiciones del Tratado de Paz" (ProInfantia, 1917:410-2).

Consecuencia de todo ello es la existencia, en el primer tercio del siglo XX, de una red internacional fraguada alrededor de congresos y asociaciones, sobre todo en Europa y América. El objetivo de la misma era atender la protección a la infancia abandonada física y moralmente, por utilizar una expresión muy extendida en una época donde los derechos de los niños y niñas eran desconocidos. Evidentemente estas actividades continuarán hasta la actualidad. Nos ha interesado poner de manifiesto esta situación incipiente para comprender el surgimiento de los diferentes tratados sobre los derechos del niño, que estudiaremos a continuación, y el papel que todavía juegan este tipo de asociaciones, ahora caracterizadas como ONG en la mayoría de los casos.

\section{Los Tratados Internacionales sobre Derechos del Niño}

\section{La Declaración de Ginebra de 1924}

Todos estos antecedentes nos explican de alguna manera la situación que precedió a la primera declaración sobre los derechos de los niños y niñas, la denominada Declaración de Ginebra, aprobada por unanimidad, en la Sociedad de Naciones en $1924^{6}$ y también el "humus" ideológico de una época. En el contexto en el que se produce esta declaración 
debemos tener presente, dentro del ámbito del derecho internacional, unos factores internos (el impulso de su fundadora, la ayuda de Benedicto XV y el apoyo de políticos e intelectuales de todo signo, como Robert Cecil uno de los constructores de la Sociedad de Naciones, y premio Nóbel de la Paz en 1957) y otros externos, como el tratado de Versalles, después de la primera guerra mundial, la Carta de Lady Aberdeen, elaborada por el Consejo Nacional de la Mujer, la Carta socialista sobre el trabajo de los adolescentes y las indicaciones de la Organización Internacional del Trabajo sobre el trabajo infantil.

Esta Declaración es muy sucinta, observándose una serie de derechos dentro de la dimensión protectora: alimento, cuidado, ayuda, acogida y socorro (título 2); educación (2, 4 y 5); reinserción del niño delincuente (2) y protección en caso de peligro (3). Todo lo cual supone un "esbozo" de la integridad de los derechos del niño, si bien no se recoge ni el derecho de los niños a unos padres ni tampoco él mismo es considerado como sujeto de derecho. Esta declaración ha sido criticada desde el punto de vista de la técnica jurídica (Garibo, 2004:85), aunque se aprecia la fundamentación del derecho de los niños al desarrollo de su personalidad. Así pues, estos derechos están planteados desde una nueva ética a favor de la infancia. El "debe ser" acoge todos los ámbitos de la vida infantil, desde el cuidado biológico, psicológico, al judicial y educativo, inspirándose todavía en una mentalidad de ayuda y protección, más que en la de considerar a los niños y niñas como sujetos de derecho. Se trata, por lo tanto, de un antecedente, que sobrevivió a la propia Sociedad de Naciones y que será referencia constante a la hora de redactar los sucesivos tratados internacionales. Desde la perspectiva histórica, y a la vista de los impulsores y de los contenidos de esta declaración, cabe afirmar que la misma debería insertarse en una consideración de los derechos de los niños dentro de las políticas del filantropismo social, característico de la época. Personajes como G. Jebb o J. Korczack, pioneros en la defensa de los derechos de la infancia, están íntimamente relacionados en su biografía con la educación y la protección a la infancia. Esta declaración fue traducida a cerca de 40 idiomas y recibió tanto adhesiones personales como institucionales ${ }^{7}$. Por otra parte, una de las labores más importantes fue la elaboración, durante tres años, de informes a nivel mundial sobre el bienestar de los niños; siendo un precedente de los que años más tarde publicará el UNICEF hasta la actualidad.

Pero esta declaración sería también el germen de otra serie de declaraciones, cartas, tablas, etc. que durante el periodo comprendido entre la misma y la siguiente, en 1959, se llevarían a cabo ${ }^{8}$. Se trata de un periodo fructífero donde se van perfilando los derechos del niño, añadiendo y sistematizando estos derechos específicos. En todos estos documentos se aprecia una percepción de la infancia regida por el desarrollo de la personalidad de los niños y niñas y la atención a sus necesidades. El valor educativo de los mismos también está presente, desde una perspectiva de innovación pedagógica.

En los años inmediatamente posteriores a la Declaración de Ginebra se observa una mayor actividad por parte de la Sociedad de Naciones, bien sea a través de la "Comisión consultiva de la trata de mujeres y niños", a partir del Convenio de 1921, bien a través de la "Comisión de protección de la Infancia y de la Juventud", donde en diversas sesiones se tratan temas relacionados con la protección de la vida y la salud, la edad de matrimonio, trabajo infantil, asistencia y repatriación de niños de nacionalidad extran- 
jera, educación, infancia abandonada y delincuente, o "los efectos del cinematógrafo sobre la mentalidad y la moralidad de los niños", tema muy de actualidad en su momento. Entre los proyectos desarrollados por la Sociedad de Naciones cabe citar un "Convenio internacional para reintegrar en sus hogares a los niños y adolescentes". En este convenio aparece un concepto que en posteriores tratados se recogerá como el "interés superior del niño", expresado de la siguiente manera: "En cuestión de asistencia, el interés del menor debe prevalecer ante todo y sobre todo" (Pro-Infantia, año XXII, 1930: 16).

También es de destacar la celebración de diferentes congresos de importancia internacional, siguiendo la línea señalada anteriormente. Nos referimos al Primer Congreso General del Niño celebrado en Ginebra en agosto de 1925, organizado alrededor de dos secciones: Higiene y Medicina y Asistencia y Previsión Social (Pro-Infantia, tomo XXIII, 1925:174). A este congreso asistieron médicos, sociólogos, pedagogos, directores de obras sociales, educadores, etc. Entre las conclusiones se resalta la importancia de que las naciones asuman las responsabilidades que les corresponde en la protección a la infancia, creando además una Oficina de Información que recogiese las informaciones remitidas por los diferentes países sobre la situación de la infancia. Así mismo, se acuerda remitir sus conclusiones a la Sociedad de Naciones. También la Asociación Internacional para la Protección de la Infancia continuará con la celebración de sus congresos, como el quinto lo celebró en Roma en 1926, y el sexto en Milán en 1928. Las secciones de estos congresos obedecía a dos ámbitos diferenciados: Sección de Higiene/Medicina y Sección Jurídica. Al igual que en el congreso anterior, en estos insisten en que "el estado debe auxiliar pecuniariamente a las instituciones privadas de beneficencia" o en que en los países en donde existan tribunales para niños se hagan estudios sobre los niños o en que haya establecimientos de educación y reforma para el correcto cumplimiento de la misión encomendada (Pro-Infantia, tomo XXVII, 1930: 7-12). También en París, en julio de 1928, siguiendo al celebrado en 1926 en Madrid, se celebró otro Congreso internacional sobre protección a la infancia, presidido por el Ministro de Trabajo e Higiene francés y que tuvo un enorme éxito tanto por la presencia de los ponentes y las entidades colaboradoras (prácticamente estuvieron presentes las más importantes asociaciones de protección a la infancia), como por la cantidad de asistentes.

\section{La Declaración de los Derechos del Niño de 1959}

Tiene que llegar el desastre de la Segunda Guerra Mundial para que, tras la Declaración Universal de los Derechos Humanos de 1948, cuyo objetivo principal era luchar por la paz, volvamos a recuperar el sendero de los derechos de los niños y niñas. El artículo 2 de esta declaración, sobre igualdad y no discriminación, será uno de los principios más importantes para el desarrollo de toda una serie de pactos y convenios internacionales posteriores. La Declaración de los Derechos del Niño de 1959 surge en un contexto internacional mas pacificado, con la recuperación de todos los países que intervinieron en la guerra y también con la puesta en marcha de los procesos de descolonización de los países africanos, reivindicando su derecho a la libre determinación, aunque estamos en un contexto de guerra fría. La creación del UNICEF en 1946, y la nueva situación de la infancia después de la Segunda Guerra Mundial, explica la nueva Declaración, 
aprobada por la Asamblea General de Naciones Unidas el 20 de noviembre de 1959. Las aportaciones más importantes en la confección de este texto serán la Declaración sobre Derechos Humanos de 1948 y el proceso de elaboración subsiguiente de los pactos de derechos civiles y políticos y de los derechos económicos, sociales y culturales, aprobados en 1966, pero que tuvieron un largo proceso de gestación y no entraron en vigor hasta 1976. De manera que desde 1949 hasta 1959 se estaría a vueltas con la nueva declaración sobre los derechos de los niños, manifestándose una tensión constante entre los países del bloque socialista, sobre todo la Unión Soviética y Polonia, partidarios de una convención que obligase más a los Estados, y los países bajo la égida de Estados Unidos más partidarios de una actualización de la Declaración de Ginebra.

Esta declaración, que es la primera declaración universal en el ámbito de las Naciones Unidas sobre un grupo de seres humanos, se expresa en 10 principios que amplían los derechos anteriores, aunque están mucho mejor sistematizados desde el punto de vista de la técnica jurídica. A la vista de esta declaración, y comparándola con la de Ginebra de 1924, se puede constatar que subyace el mismo espíritu con respecto a los valores éticos que deben presidir el reconocimiento de los derechos del niño, en aspectos como la educación, cuidados especiales a los niños discapacitados, la atención en primer lugar en casos de conflictos, formación en sentimientos de solidaridad y amistad entre los pueblos, no discriminación por razón de raza, nacionalidad o creencia, etc. Es decir, la mayoría de los principios recogidos en esta declaración estaban ya perfilados en la primera de 1924.

No obstante, aparecen algunas innovaciones importantes: una definición de niño (en el preámbulo); el derecho de los niños a unos padres (Principio 6); derecho a un nombre y una nacionalidad (Principio 3), lo cual presupone cierto reconocimiento de derechos civiles, aunque los redactores no fuesen conscientes de ello y, por primera vez aparece un concepto nuevo que supondrá en la Convención de 1989 un punto fundamental y controvertido en el ámbito jurídico: "el interés superior del niño". Así en el principio II se dice: "Al promulgar leyes con este fin, la consideración fundamental a que se atenderá será el interés superior del niño" y también en el principio VII se dice: "El interés superior del niño debe ser el principio rector de quienes tienen la responsabilidad de su educación y orientación; dicha responsabilidad incumbe en primer término a sus padres". Por lo tanto, en dos principios fundamentales como es el de la protección especial para "el desarrollo físico, mental, moral, espiritual y social en forma saludable y normal y el derecho a la educación, en el cual se encuentran implicados los gobiernos, la sociedad y los padres, queda claro que debe primar el 'interés del niño"”. Este planteamiento resulta innovador ya que debe estar por encima de cualquier otra consideración, abriendo la posibilidad a considerar a los niños como sujetos de derecho. No obstante, Cots (1979) al analizarlo, no le concede mayor importancia, a pesar de que muchas legislaciones comenzaron a considerarlo como un elemento positivo, lo cual puede ser interpretado como el lento proceso de asentamiento de conceptos innovadores. En la Convención todavía resultará más esclarecedora la postura con relación a este concepto, que figurará como uno de los principios generales.

$\mathrm{Al}$ igual que ocurrió en el periodo comprendido entre las dos declaraciones, a partir de 1959 se llevarán a cabo una serie de acciones encaminadas al reconocimiento de los derechos de los niños y niñas en el ámbito del derecho internacional ${ }^{9}$; demostrándonos 
que la permanencia por el reconocimiento de estos derechos ha sido una constante. La implicación de instituciones y organismos internacionales en el reconocimiento, cada vez más específico de diversos derechos humanos, ha conseguido desarrollar todo un entramado de derechos que lograrán fraguarse, de una manera conjunta, en la Convención de 1989.

\section{La Convención sobre los Derechos del Niño de 1989}

Treinta años más tarde, el 20 de noviembre de 1989, la Asamblea General de las Naciones Unidas, aprueba la Convención sobre los Derechos del Niño, que entró en vigor en septiembre de 1990, siendo el tratado internacional que más apoyo ha recibido a lo largo de la historia del derecho internacional, pues hasta la fecha tan solo Estados Unidos de América y Somalia no la han ratificado. La Convención fue minuciosamente elaborada durante diez años (1979-1989) con la colaboración de los representantes de todas las sociedades, todas las religiones y todas las culturas ${ }^{10}$. Pero esta Convención venía precedida por otras declaraciones, convenciones y pactos sobre reconocimiento de derechos civiles. En este proceso de elaboración la presencia de las ONG fue muy importante en un primer momento, sobre todo a partir de la declaración de la ONU sobre el Año Internacional del Niño celebrado en 1979 y la implicación de las ONG internacionales dedicadas a la infancia. Estas organizaciones establecieron un grupo intergubernamental, bajo los auspicios de la Comisión de Derechos Humanos de la Naciones Unidas, que había comenzado a redactar una Convención para sustituir a la Declaración de 1959. El UNICEF planteará su apoyo y su capacidad de movilización, sobre todo a partir de 1987, al reconocer la convergencia entre "la revolución en pro de la supervivencia y el desarrollo del niño y el proceso a favor de los derechos del niño" (UNICEF, 1996:64). Este organismo del Sistema de Naciones Unidas adoptará la Convención como "misión" para sus programas mundiales a partir de 1996.

La Convención recoge, en los primeros 41 artículos, los derechos humanos de todos los niños y niñas menores de 18 años que se deben respetar y proteger, y exige que estos derechos se apliquen a la luz de los principios rectores de la Convención ${ }^{11}$. Además, el hecho de que el reconocimiento de la Convención sea prácticamente universal, reforzando los derechos de la infancia, sustenta como instrumento jurídico una combinación única de virtudes, al subrayar y defender la función de la familia en la vida de los niños, a la obligación de los Estados la responsabilidad de los padres en materia de atención a sus hijos; a fomentar el respeto de la infancia, pero no a costa de los derechos humanos o de las responsabilidades de los otros; a apoyar el principio de no discriminación y, finalmente, a establecer claras obligaciones por parte de los Estados Partes (UNICEF, 1999).

Por otra parte, tenemos que señalar que, a diferencia de las dos declaraciones citadas que no obligaban a ningún Estado a cumplir con los derechos reconocidos, pues eran meras declaraciones de intenciones, sin ningún órgano de garantía sobre la aplicación de estos instrumentos internacionales, la Convención de 1989 es más vinculante y además crea el Comité de los Derechos del Niño que, aunque criticado cumple una serie de funciones muy adecuadas para ver la evolución y la puesta en práctica de la Con- 
vención. La crítica más fuerte se basa en que este Comité no tiene fuerza coercitiva con respecto a los Estados Parte, como ocurre con otros órganos de vigilancia, garantía y seguimiento de otros tratados internacionales (Dávila, 2001:88-90). Otra crítica importante es la relativa a las reservas hechas a la Convención por parte de los Estados. Hasta 1999, fecha del último documento del Comité sobre los Derechos del Niño referido a las reservas, han sido 69 los Estados Partes que han realizado algún tipo de reservas, siendo únicamente 26 los artículos, de los 54, los que no tienen ninguna reserva $^{12}$. En algunos casos, estas reservas pueden desvirtuar la propia Convención.

Ante la complejidad de todo el articulado de la Convención podemos encontrar algunos análisis en función del contenido; existiendo varios intentos de clasificación y siguiendo criterios diferentes ${ }^{13}$. Por nuestra parte, hemos realizado una clasificación, partiendo de los propios criterios que utiliza el Comité de los Derechos del Niño en sus recomendaciones para la cumplimentación de los informes de los respectivos Estados Partes, intentando ser fiel al contenido de la Convención. De esta manera, proponemos la siguiente clasificación, que está atravesada por los principios generales contenidos en los artículos 2 (no discriminación), 3 (interés superior del niños), 6 (derecho a la vida y la supervivencia) y 12 (respeto a las opiniones del niño), además del artículo 4 (efectividad de los derechos a cargo de los Estados Partes) y el artículo 5 (orientación de los padres y evolución de las facultades del niño), subrayando que la Convención es indivisible y sus artículos son interdependientes ${ }^{14}$. Así, nos parece pertinente destacar que, en función de cuatro categorías básicas, el conjunto de derechos podrían agruparse de la siguiente manera:

- Satisfacción de las necesidades básicas de la infancia: Atención salud y servicios médicos, seguridad social, nivel de vida, educación y desarrollo de la personalidad y juego (artículos 6, 24, 25, 26, 27, 28, 29 y 31);

- Garantía de los derechos civiles y políticos: Nombre y nacionalidad, libertad de expresión, de pensamiento, conciencia y religión, asociación, opinión del niño, protección a la vida privada, participación, de acuerdo con su capacidad y desarrollo (artículos, 7, 8, 12, 13, 14, 15, 16 y 17);

- Colaboración con la familia respetando sus derechos y obligaciones. Dirección y orientación de padres y madres para el ejercicio de los derechos del niño, separación de padres y madres, la reunificación familiar (preámbulo y artículos 5, 6, $9,10$ y 18$), y$

- Protección y atención a los niños y niñas en situaciones vulnerables y contra toda forma de explotación y violencia: Malos tratos, explotación laboral, tráfico de drogas, explotación sexual, venta o trata de niños, tortura y pena de muerte (artículos $19,32,33,34,35,36$ y 37) y Retención ilícita de niños, niños privados de medio familiar, adopción, niños refugiados, niños impedidos mental o físicamente, niños pertenecientes a minorías o poblaciones indígenas, conflictos armados, justicia del menor y reintegración social (artículos, 11, 20, 21, 22, 23, 30, $38,39$ y 40$)$. 
Como puede observarse, nuestra clasificación, nos permite insistir en las dos perspectivas que subyacen en todo el tratado y que, de alguna manera, recoge una tradición histórica en cuanto a los derechos de protección y los de autonomía. Esta clasificación nos servirá también para analizar el discurso proteccionista y los derechos de la infancia que realizamos en el siguiente epígrafe.

El éxito de la Convención es evidente, no sólo por ser el documento internacional que mayor número de adhesiones ha recibido, sino porque ha logrado estar presente como referencia para los cambios legislativos nacionales, además de suponer una orientación para el reconocimiento de los derechos de la infancia y de las prácticas de intervención social sobre la infancia, sobre todo en situaciones de exclusión social. Uno de los baremos más fiables para realizar un seguimiento sobre el cumplimiento de la Convención lo podemos encontrar en los trabajos realizados por el Comité, que es el organismo de seguimiento de la Convención. Hasta 1996, eran 50 los países que habían remitido sus informes al Comité y, por lo menos, 15 de ellos habían incorporado los principios de la Convención en sus constituciones nacionales y más de 35 habían elaborado nuevas leyes o reformado las existentes para adaptarse a sus criterios. Asimismo se constata que en otros países se ha creado la figura del Defensor del Niño y otras instituciones, además de las numerosas ONG. En la actualidad, esta situación ha cambiado notablemente, pues hasta 2004 fueron 62 Estados Partes los que habían remitido el segundo informe, lo cual es indicativo del cumplimiento por parte de los Estados Partes y su permanente actualización (UNICEF, 2004). No obstante, el éxito más relevante de la Convención es la expansión de una concepción de la infancia basada en los derechos humanos y de unos modelos de políticas proteccionistas que están sirviendo a los países en desarrollo.

\section{El discurso proteccionista sobre los derechos de la infancia.}

Después de haber mostrado las condiciones históricas que posibilitaron el surgimiento de los tres tratados internacionales sobre los derechos del niño, nos centraremos en el contenido de los mismos. Para ello hemos analizado las Declaraciones de 1924 y 1959 y la Convención de 1989, además de otros documentos, citados anteriormente. Con ello pretendemos señalar las continuidades y rupturas del discurso dominante sobre la protección a la infancia. Como podrá comprobarse, del análisis realizado resulta relativamente sencillo observar la permanencia de un discurso reiterativo sobre la concepción de la infancia, la actuación de los agentes implicados y, sobre todo, los ámbitos en los cuales los niños y las niñas son objeto o sujeto de derecho.

La primera cuestión que nos planteamos es la relativa a la extensión de los propios textos. Así, mientras la Declaración de Ginebra de 1924 contiene cinco principios, la Declaración de los Derechos del Niño de 1959, recoge 10, y la Convención de 1989 ya es un documento que contiene 54 artículos. No obstante, no se trata de cuantificar el contenido de los textos, sino elaborar una categoría que nos permita identificar los derechos reconocidos en cada una de ellas y, a partir de ello, poder establecer un tipo de discurso sobre la infancia, según esta visión internacional. 
Los ámbitos en los cuales se articula el discurso dominante sobre los derechos del niño y que parecen mostrar una permanencia son los siguientes, siguiendo nuestra clasificación anterior: 1) Satisfacción de las necesidades básicas, 2) La familia, 3) Asistencia y protección en situaciones vulnerables y 4) Garantizar los derechos civiles y políticos. Además de estos ámbitos, cabe identificar en todos esos documentos, tres categorías permanentes y que se van consolidando como principios inamovibles. Nos referimos al derecho a la educación, al principio de no discriminación y, finalmente, al presupuesto moral relativo a los "niños primero", que actúa como un recurso retórico, sin mayor desarrollo normativo hasta la Convención. Todas estas cuestiones quedan muy bien recogidas en el preámbulo de este último documento. Dos de estas categorías van a establecerse como "principios básicos" de la Convención: la "no discriminación” y el "interés superior del niño", además de los de derecho de participación y derecho a la vida ${ }^{15}$. Es decir, se trata de un discurso de una arquitectura sencilla, donde los factores dominantes, continúan siendo el cuidado de las necesidades básicas y la protección, características que polarizan el discurso sobre la infancia, a lo largo de todo el siglo XX.

Pasaremos a referirnos, en primer lugar, a estos tres últimos derechos, para centrarnos posteriormente en los cuatro ámbitos señalados. Por lo que respecta, a los "niños primero" la redacción del principio III de la Declaración de Ginebra es la base sobre la que se asienta el resto de documentos. Así, vemos como existe una continuidad en la redacción de este principio, aunque se irá matizando en cada uno de los mismos; pasando de "el niño debe ser el primero en recibir ayuda en tiempo de peligro" (1924) a “el niño debe ser el primero en recibir socorro en caso de calamidad" (1948) y finalmente en 1959 a "el niño debe en todas circunstancias figurar entre los primeros que reciban protección y socorro". Por supuesto, una plasmación de este principio será recogida bajo el lema "interés superior del niño", que tanta importancia adquiere en la Convención, aunque ya estaba presente en la declaración de 1959 (Dávila, 2001). El haber especificado, en esta última fecha "en todas circunstancias" permitía sin duda el tránsito a un fortalecimiento de ese principio, que finalmente es un "principio básico" en la Convención en su artículo 3, donde ya ese principio moral debe regir y obligar a las instituciones públicas y privadas, autoridades administrativas y al propio Estado que tomará las medidas legislativas y administrativas necesarias.

Por lo que respecta a la "no discriminación", ya en la declaración de 1924 aparece este principio, aunque no en el articulado pero sí en su preámbulo, donde se afirma que los derechos que se reconocen están por encima de "toda consideración de raza, nacionalidad o credo". En la relectura realizada por la Unión Internacional de Protección a la Infancia en 1948, esta afirmación pasa a ser el primer artículo, donde se dice que "el niño debe ser protegido excluyendo toda consideración de raza, nacionalidad o creencia”. A partir de este momento y hasta la redacción final de la declaración de 1959, este principio va sufriendo una serie de transformaciones, añadiéndose motivos de no discriminación. Así, en el documento de 1948 de la Organización Mundial de la Salud, se añaden los siguientes motivos de no discriminación: "opiniones políticas, condición económica y social". Lo mismo ocurre con el documento de la Comisión de Asuntos Sociales de 1950, donde se enumeran los siguientes: "raza, color, sexo, idioma, casta, religión, opinión 
política o cualquier otra, origen nacional o social, posición económica, legitimidad o cualquier otra situación". La redacción final de la declaración de 1959 declara que "estos derechos serán reconocidos a todos los niños sin excepción alguna, ni distinción o discriminación por motivos de raza, color, sexo, idioma, religión, opiniones políticas o de otra índole, origen nacional o social, posición económica, nacimiento u otra condición ya sea del propio niño o de su familia". Este último motivo de no discriminación se refería sin duda a la inclusión de los niños nacidos fuera del matrimonio, cuestión que era mucho más explicita en el proyecto. Por supuesto, la Convención recogerá este principio en su artículo segundo, como no podía ser de otra manera, de acuerdo con todos los tratados de derechos humanos y lo hace de una forma muy amplia; incluyendo "los impedimentos físicos, el nacimiento y cualquier otra condición, de sus padres o de sus representantes legales", además de implicar a los Estados Partes en la garantía de esos derechos y la protección contra toda forma de discriminación.

Un aspecto a reseñar en los documentos analizados, es la unión que se establece entre este principio de no discriminación y la educación. De esta manera, y ya desde la Declaración de Ginebra, se concibe que la educación ha de ser el mejor antídoto contra la discriminación, sobre todo combinando el desarrollo personal y la dedicación y servicio a los otros. De una forma paulatina se va destilando esta concepción que, finalmente, el texto de la Comisión de Asuntos Sociales de 1950 recogiendo lo dicho por la Declaración de Derechos Humanos de 1948, lo orientará hacia el desarrollo de "un espíritu de fraternidad y paz universales". Lo mismo hará el proyecto de 1959 de una manera farragosa, aunque en la redacción final del principio X de la Declaración de 1959, quede perfectamente establecida esta relación entre no discriminación y los objetivos de la educación, encaminados a un "espíritu de comprensión, tolerancia, amistad entre los pueblos, paz y fraternidad universal, y con plena conciencia de que debe consagrar sus energías y aptitudes al servicio de sus semejantes". Toda esta argumentación no puede ser olvidada cuando leemos el artículo 29 de la Convención, donde aparecen los objetivos de la educación: desarrollo de la personalidad, respeto a los derechos humanos; respeto a los padres, a la identidad cultural y valores nacionales y de otras civilizaciones; asunción de una vida responsable en una sociedad libre con respeto a la diversidad y, finalmente, respeto al medio ambiente natural.

El derecho a la educación también es un eje que atraviesa todos los documentos que estamos analizando, si bien hasta 1950, con el texto de la Comisión de Asuntos Sociales, no toma cuerpo como auténtico derecho, indicando que la educación "deberá ser gratuita", pues en los anteriores se insiste en que el "niño debe ser educado" especificando posteriormente los objetivos de la educación, pero no reconociendo la educación como un derecho. Hay que tener en cuenta que hasta la Declaración de Derechos Humanos de 1948 no se recoge explícitamente este derecho. Así pues en la Declaración de 1959, se cita textualmente que "el niño tiene derecho a recibir educación, que será gratuita y obligatoria por lo menos en las etapas elementales". A partir del Pacto de los Derechos Económicos, Sociales y Culturales de 1966, en su artículo 13, este derecho ya queda asentado de una manera definitiva. De esta manera, la Convención, en su artículo 28, lo fija de una forma que compromete a los Estados parte a asegurar el disfrute de este derecho (Beiter, 2006; Tomasevski, 2004 y 2006 y Dávila y Naya, 2005). 
Por lo que respecta a los diferentes ámbitos que hemos señalado, la satisfacción de las necesidades básicas, es sin duda un eje presente en todos los documentos. Es cierto que en los primeros textos, tan solo se apunta la dirección por dónde deben ir estas atenciones, para llegar a 1989 a una concepción amplia y bien articulada. Así en 1924, tan solo se menciona que "se debe dar a los niños los medios para su normal desarrollo, tanto material como espiritual”. En las sucesivas redacciones se irán añadiendo otros aspectos del desarrollo, como el moral, social, intelectual, etc. hasta llegar a la declaración de 1959 donde, además de recoger todos estos, añade para el disfrute de los mismos en "condiciones de libertad y dignidad", apareciendo por primera vez la mención al "interés superior del niño" como consideración fundamental a la hora de promulgar leyes. También hay que señalar que en este proceso se recogerá un derecho, único y propio de los niños y niñas como es el disfrute del juego, que no aparece en los documentos hasta 1948 y, posteriormente, en la declaración de 1959. Todos estos derechos quedarán plasmados en la Convención.

En relación directa con el desarrollo armonioso de los niños y niñas, la familia será otro ámbito que irá adquiriendo una presencia cada vez mayor. Así, mientras en la Declaración de Ginebra no se dice nada al respecto, en la nueva redacción de 1948 de la Unión Internacional de Protección a la Infancia, el principio II se refiere a que "el niño debe ser ayudado, respetando la integridad de la familia". No obstante, ya en los textos posteriores se matiza esta situación, señalando que "si el niño no tiene una vida familiar normal, la colectividad tendrá que procurarle un hogar", como cita la Organización Mundial de la Salud en 1948, y los siguientes documentos, donde se implica a la sociedad o a las autoridades públicas en aquellos casos de niños sin familia. También se procurará no separar a los niños de corta edad de su madre, e incluso se prevé que, en casos de familias numerosas, el Estado conceda subsidios a las mismas. En la Convención, en contra de lo que algunas lecturas apresuradas manifestaron en un principio, este ámbito figura como uno de los ejes fundamentales, junto con el Estado para garantizar y proteger los derechos de los niños y niñas.

Junto con todos estos derechos, tenemos que resaltar que donde los documentos adquieren mayor fuerza es en el ámbito de la asistencia y protección en situaciones vulnerables. En este sentido, los planos que cabe incluir en este ámbito son los relativos a los niños y niñas con necesidades especiales, a la protección en casos de explotación y a la asistencia y seguridad social. La Declaración de Ginebra, en su II principio, señala de una forma amplia las posibilidades de este derecho de protección, que se irá depurando hasta la declaración de 1959. Resulta ilustrativo el cambio de palabras que va sufriendo este derecho, de manera que casi podemos asistir a la propia evolución de los conceptos en relación con este tipo de población. Así se pasa de "delincuente", "deficiente", "huérfano" o "abandonado" en 1924, a "desadaptado", "inadaptado" en 1948, y de aquí a “inadaptado física, mental o socialmente" para llegar a la declaración de 1959, donde se habla de "el niño físicamente o mentalmente impedido o que sufra algún impedimento social". Lo mismo ocurre si nos referimos a la protección con respecto a todo tipo de abandono, crueldad o explotación, donde la cuestión que subyace se refiere principalmente a la explotación laboral, como finalmente señala el principio IX de la declaración 
de 1959, recordando que "no deberá permitirse al niño trabajar antes de una edad mínima adecuada...que pueda perjudicar su salud o educación". Junto con estos dos planos, el poder beneficiarse de una seguridad y previsión social es otro aspecto que subyace en estos derechos de protección. La Convención le dedicará a todo este tipo de atenciones una serie de artículos relacionados con malos tratos, explotación laboral, tráfico de drogas, explotación sexual, venta o trata de niños, tortura y pena de muerte; así como la retención ilícita de niños, niños privados de medio familiar, adopción, niños refugiados, niños impedidos mental o físicamente, niños pertenecientes a minorías o poblaciones indígenas, conflictos armados, justicia del menor y reintegración social.

Finalmente, y con relación al reconocimiento de los derechos civiles y políticos, hay que recordar que éstos tendrán una entrada tardía en el campo de los derechos del niño y como consecuencia de la Declaración de Derechos Humanos de 1948. Así en el documento de la Comisión de Asuntos Sociales de 1950, aparece por primera vez el derecho que les asiste a los niños a un nombre y una nacionalidad, conservando la misma redacción tanto en el proyecto como en la declaración de 1959. No obstante, y como se ha señalado tantas veces, una de las novedades mayores de la Convención es la apertura a los derechos de autonomía que han dado lugar a una concepción diferente de los derechos del niño, pasando a ser sujeto de derecho. En este sentido, estos derechos se especificarán no sólo en el de nombre y nacionalidad, sino también en los de libertad de expresión, de pensamiento, conciencia y religión, asociación, opinión del niño, protección a la vida privada, participación, de acuerdo con su capacidad y desarrollo.Por lo tanto, podemos apreciar que el discurso sobre la infancia durante el Siglo XX mantiene una continuidad basada, sobre todo, en la defensa de dos ámbitos de derechos diferenciados, uno referente a la protección y la satisfacción de necesidades básicas y otro a los derechos de autonomía de los niños y niñas. De esta manera podemos afirmar la continuidad de un discurso proteccionista de la infancia; cuya ruptura mayor se produjo en 1989 con el reconocimiento de los derechos civiles y políticos de los niños y niñas, sujetos ya de derecho. 


\section{Referencias}

Barona, J.L. (2004). El Consejo Superior de Protección a la Infancia y Represión de la Mendicidad (1904-1914). Su ideología social y sanitaria in Perdiguero, E. Salvad al niño. Valencia: Seminari d'Estudis sobre la Ciència, 121-155.

Beiter, K. D. (2005). The Protection of the Right to Education by International Law. Leiden/Boston: Martinus Nijhoff Publishers.

Calvo, M. (2003). Implementación de los Derechos del Niño, in Soroeta, J. (ed.): Cursos de derechos humanos de Donostia-San Sebastián Vol. 4. Bilbao: Servicio Editorial de la Universidad del País Vasco.

Cots, J. (1979): La declaració universal dels drets de l'infant. Barcelona: Rosa Sensat- Ediciones 62.

David, P. (1997). Reflexiones sobre el trabajo del Comité de las Naciones Unidas sobre los Derechos del Niño. La Convención sobre los Derechos del Niño: se reta a los gobiernos de Europa Occidental in FUNCOE: El futuro de la infancia en Europa. Actas del Seminario Europeo la Protección de los niños y niñas y de las familias: el papel de las ONG en la UE. Madrid: FUNCOE, 79-103.

Dávila, P. (2001). Los derechos de la infancia, UNICEF y la educación, in Naya, L.M. (Coord.): La educación a lo largo de la vida, una visión internacional. Donostia: Editorial Erein, 61-117.

Dávila, P. y Naya, L.M. (2005). Infancia y educación en el marco de los Derechos Humanos in Naya, L.M. (coord): La educación y los derechos humanos. Donostia: Erein, 91-135,

Dávila, P. y Naya, L.M. (Ed.) (2005). La Infancia en la Historia: espacios y representaciones, San Sebastián: Erein, 2 vol.

Detrick, S. (1992). The United Nations Convention on the Rights of the Child. A guide to the "Travaux Préparatoires". Dordrecht/Boston/London: Martinus Nijhoff Publishers.

Donna J. G. (1988). The Pan American Child Congresses, 1916-1942: Pan Americanism, child reform, and welfare state in Latin America Journal of Family History, 23 (3), 272-291

Fass, P.S. (2004). Encyclopedia of Children and Childhood in History and Society, Farmington Hill, MI: The Gale Group, 4 vol.,

Garibo, A. P. (2004). Los derechos de los niños: Una fundamentación. Madrid: Ministerio de Trabajo y Asuntos Sociales.

Hierro, L. L. (1999). Los derechos humanos del niño, en Marzal, A. Los derechos humanos del niño, de los trabajadores, de las minorías y complejidad del sujeto. Barcelona: Bosch-Esade, (15-32).

Hodgkin, R. y Newell, P. (2001). Manual de Aplicación de la Convención sobre Derechos del Niño, Ginebra: UNICEF. Segunda edición ampliada en 2004.

Rollet, C. (2001). La santé et la protection de l'enfant vues à travers les congrès internationaux (1880-1920). Annales de Démographie Historique, (1), 97-116.

Suarez Sandomingo, J.M. (1999). Historia dos dereitos da infancia. Santiago de Compostela, Xunta de Galicia.

Therborn, G. (1993). Los derechos de los niños desde la constitución del concepto moderno de menor: un estudio comparado de los países occidentales in Moreno, L. Intercambio social y desarrollo del bienestar. Madrid: Consejo Superior de Investigaciones Científicas.

Tomasevski, K. (2004): El asalto a la educación. Barcelona: Intermon Oxfam.

Tomasevski, K. (2006): Human Rights Obligations in Education. The 4-A Scheme. Nijmegen (The Netherlands): Wolf Legal Publishers.

Trinidad, P. (2003). El 'Talón de Aquiles' de la Convención de los Derechos del Niño: una aproximación a las Declaraciones y Reservas formuladas por los Estados Partes, in Soroeta, J. (ed.) (2003). Cursos de derechos humanos de Donostia-San Sebastián Vol. 4. Bilbao: Servicio Editorial de la Universidad del País Vasco.

UNICEF (1996). Cincuenta años en pro de la infancia in Estado mundial de la Infancia 1996. Ginebra: UNICEF. 
UNICEF (1999). La convención sobre los derechos del niño. 10 Aniversario. Ginebra: UNICEF. UNICEF (2004). Study on the impact of the implementation of the Convention on the Right of the child, Firenze: Innocenti Research Centre.

Verhellen, E. (1999): Apoyar los derechos educativos del niño: expectativas y demandas de docentes y de padres, Perspectivas, XXIX (2), junio, 245-254.

\section{Anexo I \\ Congresos Internacionales dedicados a la infancia}

\section{Protección a la Infancia}

1883, Congreso internacional de protección de la infancia, París, 15-23 junio

1890, Congreso Internacional para el estudio de cuestiones relativas a la protección de los detenidos y de los niños moralmente abandonados, Anvers, 9-14 octubre

1895, Congreso Internacional de Protección de la Infancia, Burdeos, 29-julio-3 agosto

1896, Congreso Internacional de Protección de la Infancia, Ginebra, 14-19 septiembre

1896, Congreso Internacional por la Infancia, Florencia, 5-10 octubre

1899, Congreso Internacional por la Infancia, Budapest, 13-17 septiembre

1902, III Conferencia Internacional para la protección de la infancia, Londres, 15-18 julio

1904, Congreso Internacional por la Infancia, Londres

1905, IV Congreso Internacional para el estudio de cuestiones relativas a la protección de los condenados, de los niños moralmente abandonado, vagabundos y enajenados, Lieja, 8-12 agosto

1908, Congreso Internacional por el Bienestar del niño, Washington, 10-17 marzo

1909, Congreso Regional, Pro-Infantia, Italia

1911, I Congreso Internacional de Tribunales para niños, Francia

1911, Congreso Internacional para el estudio de cuestiones relativas a la protección de los liberados y de los niños moralmente abandonados y a obras de protección, Anvers, 16-21 julio

1912, II Congreso Nacional de Protección a la Infancia, Turín

1913, I Congreso Internacional para la protección de la infancia, Bruselas, 23-26 julio

1913, Congreso Internacional de Protección a la Infancia, Bélgica

1913, Congreso Internacional para la protección de la infancia en Bruselas, 23 a 26-7

1913, IX Congreso Nacional Francés de la Asociación Católica de las Obras de Protección de la Joven

1913, I Congreso Nacional del Niño, Buenos Aires, mayo

1913, III Congreso Alemán de los Tribunales para niños, 10-12 de octubre

1914, II Congreso Penitenciario Español, 1-10 agosto

1914, Congreso Protector, Berna

1919, Congreso Internacional de la Infancia, Montevideo

1920, IV Congreso Internacional de Protección a la Infancia, Londres, 20-25 de septiembre

1922, Congreso Internacional de protección maternal e infantil, París 6-8 de julio

1924, I Congreso General del Niño, Ginebra, agosto

1926, V Congreso de la "Association Internacionale pour la protection de l'Enfance",

Roma, mayo

1927, VI Congreso de la "Association Internacionale pour la protection de l'Enfance",

Milán

1929, X Congreso Mexicano del niño, México

1930, Congreso Internacional de Protección a la Infancia, Lieja, 31-7 a 4-8

1930, Congreso Penal y Penitenciario, Praga 
Medicina (Pediatría, Salud y Gotas de Leche)

1895, Congreso Internacional de Ginecología, Obstetricia y Pediatría, París

1898, Congreso Periódico de Ginecología, Obstetricia y Pediatría, Marsella

1904, Congreso Periódico de Ginecología, Obstetricia y Pediatría, Rouen

1905, I Congreso Internacional de Gotas de Leche y de protección de la primera infancia,

París, 20-21 octubre

1907, II Congreso Internacional de Gotas de Leche y de protección de la primera infancia,

Bruselas, 12-16 septiembre

1909, Congreso Antialcohólico, Inglaterra

1910, Francia, Congreso de Practicantes

1911, III Congreso Internacional de Gotas de Leche y de protección de la primera infancia, Berlín, 11-15 septiembre

1911, Italia, Congreso de la Sociedad Italiana de Pediatría

1911, Se celebran dos congresos, uno en Burdeos y otro en Lyon en Octubre, en ambos hay una sección especial médicos y profesionales de la instrucción. En Lyon se trata más la cuestión médica y en Burdeos la pedagógica

1912, I Congreso Internacional de Pediatría, París, 7-9-octubre

1912, VI Congreso Internacional de Ginecología y Obstetricia, Berlín, 9-13 septiembre

1913, Congreso alemán para la educación infantil antialcohólica, del 26 al 28 de mayo

1914, Congreso de Pediatría, Palma abril

1914, Congreso Internacional contra el alcoholismo, Milán, 22-9

1915, LXV Congreso de la Asociación de Médicos Americanos

1916, Congreso Anual de odontología, Suiza

1917, I Congreso Argentino de Medicina

1930, IICongreso Internacional de Pediatría, Estocolmo

\section{Educación}

1905, I Congreso de educación y protección de la infancia en la familia y de la Federación de padres y educadores, Lieja, 18-20 septiembre

1906, II Congreso de educación y protección de la infancia en la familia, Milán, 2-5 septiembre

1910, II Congreso Internación de educación y protección..., Bruselas, 21-25 agosto

1910, Congreso Pedagógico, Bruselas

1910, Congreso de Educación Familiar, Bruselas 21-25 de agosto

1911, II Congreso Internacional de Educación Moral, Holanda (Suspendido por la guerra)

1911, II Congreso Nacional de Colonias de Vacaciones, Francia

1911, VIII Congreso Nacional a favor de la educación de los niños débiles de espíritu o idiotas, Suiza

1913, Congreso Internacional de Educación Física, París 17-20 mayo

1913, II Congreso Internacional de Enseñanza del Hogar

1914, Congreso Internacional de Educación Familiar, Filadelfia, 29-9 a 2-10

1922, II Congreso Internacional de Educación Moral, Ginebra, 28-7 a 1-8

1924, Congreso Internacional de Enseñanza técnica, 27-29 de septiembre

1925, Congreso Internacional de Orientación Profesional, Burdeos, 23-26 de septiembre 


\section{Notas}

1 Este artículo se enmarca en una investigación financiada por el Ministerio de Educación y Ciencia, número de proyecto SEJ2004-01690/EDUC

2 Para conocer el caso español puede consultarse, entre otras obras: Morales, J. L. (1969) El niño en la cultura española, Madrid: Edición del propio autor. Historia de la Educación, número 10 (1991) y 18 (1999), dedicados a la Historia de la Educación Infantil e "Historia de la Educación Social" respectivamente; Chust, T. (1968). Historia de la obra de los Tribunales Tutelares de Menores en España, Madrid: Consejo Superior de Protección de Menores; Ruiz, C. y Palacios, I. (1995). Pauperismo y educación. S. XVIII-XX, Valencia: Universidad de Valencia; Ramas Varo; Ma L. (2001). La protección legal de la infancia en España. Madrid, Consejo Económico y Social; Ruiz Rodrigo, C. (2004). Protección a la infancia en España. Reforma social y educación, Valencia: Universidad de Valencia; Santolaria, F. (1997). Marginación y educación, Barcelona: Ariel; Corts, I. (2001). "Los derechos de la Infancia en España. Una perspectiva histórica” en Llorent, V. Derechos y educación de niños y niñas un enfoque multicultural, Sevilla: Departamento de Teoría e Historia de la educación, pp. 83-101; Borrás Llop, J.M. (1996). Historia de la Infancia en la España contemporánea 1834-1956, Madrid: Ministerio de Trabajo y Asuntos Sociales.

3 Para un conocimiento exhaustivo de la misma puede consultarse el número dedicado a "Enfances" de la revista Annales de Démographie Historique, (2001), no 2, p. 5-100.

Disponible en http://www.cairn.be/revue.php?ID_REVUE=ADH. En el comentario sobre el periodo contemporáneo, Catherine Rollet, demógrafa de prestigio internacional, se refiere a "les droits de l'enfant" para reseñar aquella bibliografía más directamente relacionada con los derechos del niño, huyendo de otras clasificaciones tradicionales. Por otra parte, no deja de ser curioso que una obra tan voluminosa como la de Fass, P.S. (2004). Encyclopedia of Children and Childhood in History and Society, Farmington Hill, MI: The Gale Group, 4 vol., dedique muy poco espacio a este tema. También puede consultarse Dávila, P. y Naya, L.M. (Ed.) (2005). La Infancia en la Historia: espacios y representaciones, San Sebastián: Editorial Erein, 2 vol., obra en la que se recogen las actas del XIII Coloquio de Historia de la Educación, con especial referencia al caso español y latinoamericano.

4 Para conocer la evolución de estos congresos, hemos utilizado la información que facilitaba la revista Pro-Infantia, pues nos parece que es una fuente interesante, no sólo para conocer los intereses del Consejo Superior de Protección y Represión de la Mendicidad de quien dependía, sino para tener acceso a la información que se producía en este ámbito en el resto del mundo. Además este Boletín ofrecía importante información sobre las leyes, proyectos y temas de interés sobre la protección a la infancia. En algunos casos se publican extensos comentarios por parte de los representantes españoles, como Tolosa Latour, Pulido, etc. En este sentido, es de destacar la cantidad de información a la que tienen acceso, o las buenas relaciones de los representantes españoles con el resto de colegas europeos, que se plasma en los comentarios elogiosos y el conocimiento personal. En la tabla anexa figura la relación de congresos internacionales de los que da noticia o comentario la citada revista. Para un conocimiento del funcionamiento de este consejo, puede consultarse Barona, J.L. (2004). El Consejo Superior de Protección a la Infancia y Represión de la Mendicidad (1904-1914). Su ideología social y sanitaria in Perdiguero, E. Salvad al niño. Valencia: Seminari d'Estudis sobre la Ciència, pp. 121-155. Han sido revisados los números de Pro-Infantia del citado período con la excepción de los años 1912, 1918, 1920 y 1921 dado que ha sido imposible su localización.

5 Tenemos que tener presente, además, los Congresos Panamericanos del Niño iniciados en Buenos Aires en 1916, y que tendrán su continuación en las capitales de los siguientes países Uruguay (1919), Brasil (1922), Chile (1924), Cuba (1927), Perú (1930), México (1935), Estados Unidos (1942), Venezuela (1948) y que han continuado celebrándose hasta la actualidad (Donna, 1988). Nuestro interés no es hacer un seguimiento de todos lo congresos posibles, sino destacar su proyección internacional en un contexto donde 
no existía ningún organismo internacional de derechos humanos, de ahí que nos centremos en el primer tercio del siglo XX.

6 Por lo que respecta a esta declaración, hemos de señalar que el surgimiento de la primera ONG de infancia (Save the Children Fund en 1919 y Save the Children International Union en 1920) no es ajena a la misma pues elaboró esta primera declaración. Esta organización fue creada por Eglentyne Jebb (1876-1928), historiadora y maestra, y su hermana en 1919 en Londres con el objetivo de ayudar a los millones de niños refugiados y desplazados diseminados por Europa después de la Primera Guerra Mundial. Cuenta con miembros de 26 países, habiendo recibido el Premio Príncipe de Asturias de la Concordia en 1994 por su labor a favor de la infancia, es organismo consultor del Consejo Económico y Social de Naciones Unidas y Premio Nóbel de la Paz en 1997, y sus ámbitos de trabajo en la actualidad son los Derechos del Niño, pobreza y economía internacional, conflictos armados y niños desplazados y educación básica. Hemos de señalar también que esta organización trabajó a favor de la evacuación de los niños y niñas vascas durante la guerra civil española y el intercambio de niños entre los bandos enfrentados.

7 Por ejemplo, recibió el apoyo del IV Congreso Panamericano de la Infancia, celebrado en Santiago de Chile en octubre de 1924. También tendría repercusión en las recomendaciones de la Sociedad de Naciones a todos los Estados miembros. En Francia, el Ministro de Instrucción Pública ordenó que en cada escuela estuviese colgada una copia de dicha declaración; en Austria, el texto fue firmado en una ceremonia a la que acudió E. Jebb. No obstante, el caso más significativo es el de España, que incorporó esta declaración en el Art. 43 de la Constitución republicana de 1931, donde al referirse a la familia y sus obligaciones para con los hijos, introduce novedades sobre la investigación de la paternidad, etc. y que dice textualmente: "El Estado prestará asistencia a los enfermos y ancianos, protección a la maternidad y a la infancia, haciendo suya la 'Declaración de Ginebra, o tabla de los derechos del niño'”.

8 Tabla de los Derechos del Niño del Instituto Interamericano del Niño, de 9 de junio de 1927; Texto de Gabriela Mistral sobre los derechos del niño de diciembre de 1927; Carta de la Casa Blanca de 1930; Carta de la infancia para el mundo de la postguerra de 1942, adoptada por La Conferencia Internacional; Carta de la infancia en tiempos de guerra, adoptada en 1942 por la Comisión de la Infancia en tiempos de guerra de la Oficina de la Infancia de los Estados Unidos; Declaración de oportunidades para el niño, aprobada en el Octavo Congreso Panamericana del Niño, Celebrado en Washington, Estados Unidos de América, del 9 de mayo; de 1942; Declaración de Caracas sobre la salud del niño, del IX Congreso Panamericano del Niño el 7 de enero de 1948; Carta de la Unión Internacional de Protección a al Infancia, de 1948; Proyecto de Declaración de las Naciones Unidas relativo a los Derechos del Niño, propuesto por la Organización Mundial de la Salud, de diciembre de 1948; Proyecto de Declaración de Derechos del Niño de la Comisión de Asuntos Sociales del ECOSOC de 1950; Proyecto de Declaración de Derechos del Niño de la Comisión; de Derechos Humanos de 1959. Todos estos textos se pueden consultar en la obra de Garibo, A. P. (2004: 271-339). Los últimos cuatro textos serán objeto de análisis al referirnos al discurso proteccionista sobre la infancia.

9 Además de los citados pactos internacionales de 1966, podemos considerar los siguientes documentos: Convención de la UNESCO relativa a la lucha contra las discriminaciones en la esfera de la enseñanza, de diciembre de 1960; Convención sobre el consentimiento para el matrimonio, de 1962; Declaración sobre el progreso y el desarrollo en lo social, de 1969; Declaración de los derechos del retrasado mental, de 1961; Declaración universal sobre la erradicación del hambre y la malnutrición, de 1974; Declaración sobre la protección de la mujer y el niño en estados de emergencia o de conflicto armado, de 1974; Declaración de los derechos de los impedidos, de 1975; Las Reglas de Beijing para la administración de justicia de menores de 1985; Declaración sobre los principios sociales y jurídicos relativos a la protección y bienestar 
de los niños de 1986. Además de todas estas declaraciones de carácter universal debemos tener presente las dictadas por organismos regionales sean africanos, asiáticos, americanos o europeos. (Garibo, 2004: 170-176).

10 La propuesta inicial de una Convención sobre los Derechos del Niño, partió en 1978 del gobierno de Polonia que sometió a la Comisión de Derechos Humanos de las Naciones Unidas, una propuesta en este sentido. Un grupo de trabajo compuesto por dicha Comisión, expertos independientes y delegaciones de observadores de los gobiernos no miembros, organizaciones no gubernamentales (ONG) y organismos de las Naciones Unidas, se encargaron de la preparación del borrador. Las ONG que participaron en la preparación del borrador representaban un abanico de intereses que abarcaban desde las esferas jurídicas hasta el ámbito de la protección de la familia. Para un estudio sobre la gestación de la Convención, puede consultarse Detrick (1992).

11 Los artículos 42 al 45 abarcan la obligación de los Estados Partes de difundir los principios y las disposiciones de la Convención entre los adultos y los niños; la aplicación de la Convención y la verificación de los progresos alcanzados hacia el cumplimiento de los derechos de los niños mediante las obligaciones de los Estados Partes y la responsabilidad de presentar informes de los Estados Partes y, finalmente, las cláusulas finales (artículos 46 a 54) abarcan el proceso de adhesión y de ratificación de los Estados Partes; la entrada en vigor de la Convención; y la función como depositario del Secretario General de las Naciones Unidas (Dávila, 2001:80)

12 Hay que señalar que la Convención guarda silencio sobre el efecto legal que puedan tener esas reservas, cuando "podría haber indicado de forma expresa a qué disposiciones de la CDN pueden las partes formular reservas sin que ello suponga un atentado contra su objeto y fin (o al contrario haber indicado los prefectos sobre los que las reservas serían inadmisibles). En otras palabras, el Comité debería haber expresado cual es el 'núcleo duro de la Convención' si es que considera que lo hay" (Trinidad, 2003:412)

13 Por ejemplo, Garibo (2004:201-203), centrándose en los derechos de igualdad, libertad y seguridad; distingue los derechos de protección y los derechos de autonomía. También Suarez Sandomingo (1999), por su parte, realiza una clasificación de los derechos en función de una serie de conjuntos de derechos: igualdad, integridad, civiles, políticos, culturales, etc. Asimismo se puede consultar David (1997), secretario del Comité, y Verhellen (1999)

14 Para ver la interdependencia de los artículos de la Convención resulta interesante la lectura realizada por Hodgkin y Newell en su obra Manual de Aplicación de la Convención sobre Derechos del Niño, UNICEF, (2001) donde se siguen los criterios del propio Comité. En la Observación General número 5/2003, párrafo 12, el Comité de los Derechos del Niño, refiriéndose a los citados artículos señala que la "adopción de una perspectiva basada en los derechos del niño, mediante la acción del gobierno, del parlamento y de la judicatura es necesaria para la aplicación efectiva de toda la Convención, particularmente habida cuenta de los siguientes artículos [2, 3, 6 y 12] de la Convención, identificados por el Comité como principios generales"

15 Observación General no 5 (2003). Medidas generales de aplicación de la Convención sobre los Derechos del Niño, artículos 4 y 42 y párrafo 6 del artículo 44 
94 Encounters/Encuentros/Rencontres 